\section{Marfanoid-progeroid-lipodystrophy syndrome: a newly recognized fibrillinopathy}

\author{
Eberhard Passarge ${ }^{\star, 1,2}$, Peter N Robinson ${ }^{3}$ and \\ Luitgard M Graul-Neumann ${ }^{4}$
}

We review six previous reports between 2000 and 2014 of seven unrelated patients with mutations in the $F B N 1$ gene affecting function. All mutations occurred in exon 64 of the FBN1 gene. A distinctive phenotype consisting of partial manifestations of Marfan syndrome, a progeroid facial appearance, and clinical features of lipodystrophy was present in all individuals. We suggest that this previously unknown genotype/phenotype relationship constitutes a new fibrillinopathy for which the name marfanoid-progeroid-lipodystrophy syndrome would be appropriate.

European Journal of Human Genetics (2016) 24, 1244-1247; doi:10.1038/ejhg.2016.6; published online 10 February 2016

A wide range of phenotypes results from mutations affecting function of the fibrillin-1 gene FBN1 (MIM 134797), collectively called the type 1 fibrillinopathies. ${ }^{1,2}$ These include autosomal dominant Marfan syndrome (MFS; MIM 154700), isolated ectopia lentis-2 (MIM 129600), isolated ascending aortic aneurysm and dissections, neonatal MFS, WeillMarchesani syndrome-2 (MIM 608328), AD (MIM 102370), stiff skin syndrome (MIM 184900), and GD dysplasia-2 (MIM 614185).

Many FBN1 mutations overlap with classical MFS (MIM 154797), comprising ocular, cardiovascular, and skeletal manifestations as defined by the Ghent diagnostic criteria, ${ }^{3}$ but others differ from MFS. ${ }^{1}$ The UMD FBN1 database (http://www.umd.be/FBN1/) as of 28 August 2014 (accessed 20 July, 2015) contains 3077 mutations. ${ }^{4}$ That the Marfan phenotype evolves with age has been documented in 259 children with mutations affecting function of the FBN1 gene. ${ }^{5}$ A variable genotype/phenotype relationship has been observed between the type of mutation and Marfan phenotype. ${ }^{6,7}$

Six recent reports describe seven patients with a newly recognized syndrome, the clinical features of which overlap with those of congenital MFS, progeroid syndromes, and lipodystrophy. ${ }^{8-13}$ All seven individuals harbor a disease-causing mutation in exon 64 , the penultimate exon of the FBN1 gene (Table 1).

In 2010 Graul-Neumann et al described a 27-year-old female patient with clinical features of congenital lipodystrophy, a progeroid facial appearance, and some signs of MFS. ${ }^{8}$ Recognizable mutations in seven known lipodystrophy-associated genes (APGAT2, BSCL2, CAV1, LMNA, PPARG, LMNB2, and $P T R F-C A V I N)$ and two progeroid-associated genes (LMNA/C and ZMPSTE24) were ruled out by extensive molecular analysis. Additional sequencing of the coding regions of the MFSassociated genes (FBN1, TGFBR1, and TGFBR2) revealed a de novo heterozygous 2bp deletion c.8155_8156delAA in the coding exon 64 of the FBN1 gene. ${ }^{8}$ (The annotation of this and all the following FBN1 variants is with respect to NM_000138.4.) This mutation was absent in both parents, the patient's unaffected sister, and in 150 unrelated controls. Multiplex ligation-dependent probe amplification excluded an additional deletion in the other allele. The deletion predicted a

${ }^{1}$ Institut für Humangenetik, Universitätsklinikum Essen, Essen, Germany; ${ }^{2}$ Institut für Humangenetik, Universitätsklinikum Leipzig, Leipzig, Germany; ${ }^{3}$ Institut für Medizinische Genetik und Humangenetik Charité Berlin, Berlin, Germany; ${ }^{4}$ Ambulantes Gesundheitszentrum der Charité Campus Virchow, Humangenetik, Universitätsmedizin Berlin, Berlin, Germany

*Correspondence: Dr E Passarge, Institut für Humangenetik, Universitätsklinikum Essen, Hufelandstr. 55, Essen 45122, Germany. Tel: +49 2324 41603; Fax: +49 201723 5900; E-mail: eberhard.passarge@uni-due.de

Received 8 September 2015; revised 9 December 2015; accepted 16 December 2015; published online 10 February 2016

frame shift leading to a premature stop codon 17 codons downstream, p.(Lys2719Aspfs $\left.{ }^{\star} 18\right)$, resulting in a truncated fibrillin-1 protein. Clinical signs consistent with a MFS phenotype were severe myopia ( -11 diopters), lens dislocation, dilatation of the aortic root $(32 \mathrm{~mm}$ at the age of 16 years; $35 \mathrm{~mm}$ at the age of 27 years, both in the 97th percentile for body surface with otherwise normal aortic structures), and lumbosacral dural ectasia. Thus, three major criteria of the Ghent classification were fulfilled. ${ }^{3}$ The predominant clinical signs in this patient were an extreme congenital lack of subcutaneous fat tissues and a consequent progeroid appearance of her face and body (Figure 1). Normal levels of fasting glucose, fasting insulin, C-peptide, and normal insulin receptors in cultured fibroblasts excluded diabetes mellitus, insulin resistance, and glucose intolerance. How the FBN1 mutation may have contributed to the unusual phenotype remained an open question. One of us (EP) had previously followed this patient for 25 years without being able to establish a definitive diagnosis.

This first observation has been supported by five similar reports in six further unrelated individuals. ${ }^{9-13}$

In all seven patients, the mutation is located in exon 64 of the FBN1 gene, as summarized in Table 1. All were de novo; two patients carried the same splice site mutation. ${ }^{9,12}$ Garg and Xing ${ }^{13}$ excluded other disease-causing genes in their two patients by whole-exome sequencing.

The main clinical features of this newly recognized disorder, summarized in Table 2, include intrauterine growth retardation, birth before 40 weeks gestation, and generalized lack of subcutaneous fat except in the breast and iliac region leading to a senile appearance of the face at birth in all patients (Figure 1). Mental and motor development are within normal limits. Associated clinical signs of MFS are variable. Whereas hyperextensible joints, arachnodactyly, and severe myopia have been observed in 6/7 individuals, other important signs of MFS are not present in all: aortic root dilatation in $3 / 7$, mitral valve prolapse in $3 / 7$, lumbosacral dural ectasia in 2 (in 5 not recorded), pectus excavatus in $3 / 7$, and lens dislocation in 3/7. Scoliosis was reported in two patients aged 23 and 17 years, but not in the others. In view of the differences in ages ranging from 3.5 to 27 years, the clinical findings in these five patients are difficult to interpret and compare. The three manifestations of this disorder, (i) incomplete signs of MFS; (ii) progeroid appearance not associated with other manifestations of early aging; and 
(iii) lipodystrophy not associated with metabolic disturbances, appear to be limited to mutations affecting function of the FBN1 gene in exon 64 (Figure 2).

Currently, no clear genotype/phenotype relationship has been established with the exception of the so-called neonatal region in FBN1 exons 24-32 where a subset of mutations is associated with extremely severe manifestations with onset at birth (PMID: 10189088). In addition, all FBN1 mutations associated with geleophysic (GD) and acromicric dysplasia $(\mathrm{AD})$ are located in exons 41 and 42 (PMID: 21683322), and mutations found in a subset of individuals with stiff skin syndrome are all located within exon 37

Table 1 Marfanoid-progeroid-lipodystrophy syndrome-de novo mutations in exon 64 of the FBN1 gene

\begin{tabular}{|c|c|c|}
\hline \multirow{2}{*}{$\begin{array}{l}\text { Authors } \\
\text { Graul-Neumann et } a l^{8}\end{array}$} & \multicolumn{2}{|c|}{ Type of mutations and functional consequences } \\
\hline & c.8155_8156delAA & p.(Lys2719Aspfs*18) \\
\hline Horn and Robinson ${ }^{9}$ & c. $8226+1 \mathrm{G}>\mathrm{T}$ & Splice mutation, exon 64 skipping \\
\hline Goldblatt et a ${ }^{10}$ & c.8156_8175del & p.(Lys2719Thrfs*12) \\
\hline Takenouchi et a/11 & c.8175_8182del8bp & p.(Arg2726Glufs*9) \\
\hline Jacquinet et al ${ }^{12}$ & c. $8226+1 \mathrm{G}>\mathrm{A}$ & splice mutation, exon 64 skipping \\
\hline \multirow[t]{3}{*}{ Garg and Xing ${ }^{13}$} & c.8206_8026insA & p.(Thr2736Asnfs*1) (patient 1) \\
\hline & c.8222T >C & p.(Ile2741Thr ) (patient 2) \\
\hline & c. $8226+1 \mathrm{G}>\mathrm{T}$ & p.(Glu2742Glufs*43) \\
\hline
\end{tabular}

(PMID: 20375004). The clustering of FBN1 mutations found in individuals with the progeroid form suggest the existence of a fourth substantial genotype/phenotype relationship for some FBN1 mutations. phenotype in the condition considered here raises an interesting question: How can mutations in only one exon lead to such a remarkable pleiotropic phenotype with some variable MFS signs, but otherwise different from classical MFS? The mutations reported to date in the progeroid form of MFS lead to frame shifts and premature truncation codons that are predicted not to be subjected to $\mathrm{NMD}$, the mutations being either small
The relationship between genotype and

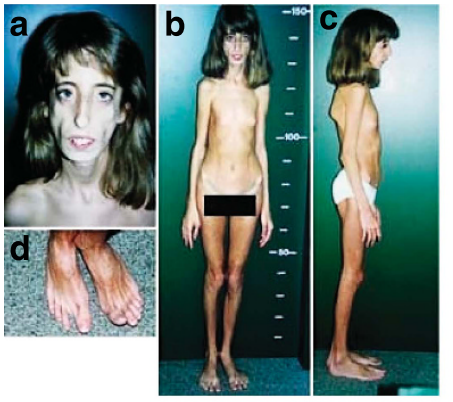

(Graul-Neumann et al, 2010 ${ }^{8}$ )

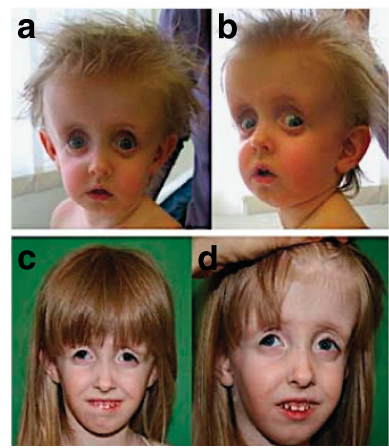

(Horn \& Robinson, $2011^{9}$ )
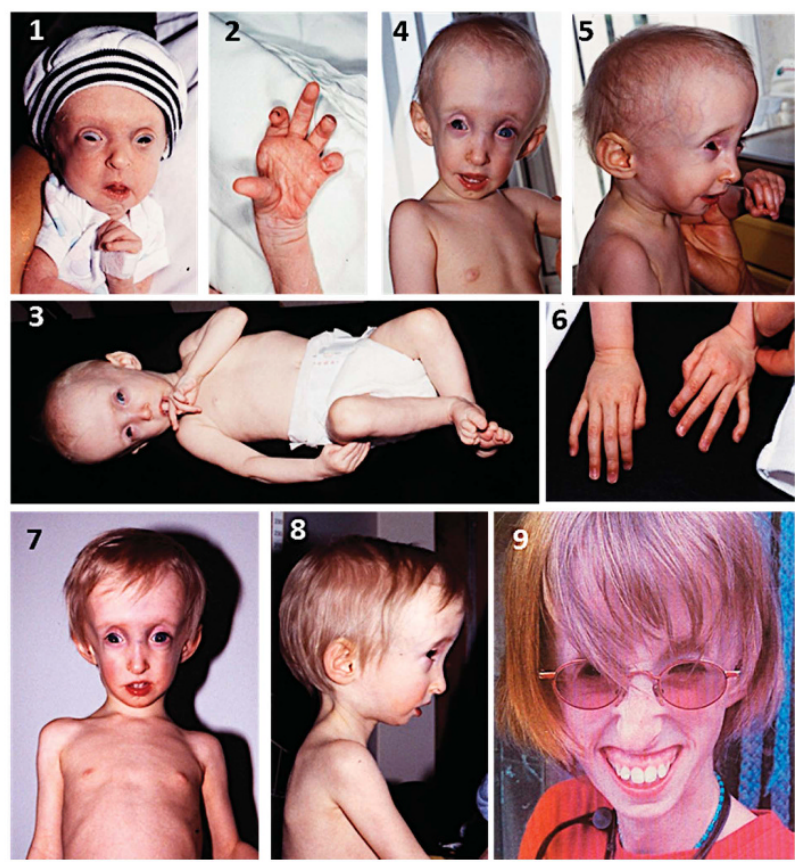

(Jacquinet et al, 2014 ${ }^{12}$ ) insertions or deletions in coding exon 64 or alter the donor splice site of intron 64 . Fibrillin-1 consists of 2871 amino acids and contains multiple epidermal growth factor (EGF)-like domains with both calciumbinding (cbEGF) and non-calcium-binding (EGF) properties that interact with other proteins. ${ }^{14}$ It results from proteolytic cleavage of profibrillin-1 at a cleavage site between amino acids arginine 2731 and serine 2732 in exon 64 , corresponding to codons 2685-2742 (nt 8052-8226), at the end of the carboxyterminal domain. ${ }^{15}$ Three groups of fibrillins, as $350-\mathrm{kDa}$ extracellular matrix proteins together with transforming growth factor $\beta$ binding proteins, are important components of the extracellular matrix. ${ }^{2,15-17}$ Jacquinet et $a l^{12}$ noted that exon 64 of profibrillin-1 harbors a highly conserved recognition sequence R-G-R-K-R-R for propeptidase convertases of furin. ${ }^{17}$ Defective microfibril function will affect the formation and organization of fibrillin monomers within microfibrils, and interfere with the structure and function of the extracellular matrix in general. As the C-terminal globular domain of fibrillin-1 is structurally similar to other extracellular matrix proteins, fibulin-3 and fibulin $4,{ }^{18,19}$ the functional loss of this segment might be critical for the pathogenesis of

Figure 1 Clinical phenotype of marfanoid-progeroid-lipodystrophy syndrome in three unrelated individuals (from Graul-Neumann et al, 2010, ${ }^{8}$ Horn \& Robinson, 2011,9 and Jacqinet et al, 2014 ${ }^{12}$ ). 
Table 2 Main clinical features of Marfanoid-progeroid-lipodystrophy syndrome

\begin{tabular}{|c|c|c|c|c|c|c|c|}
\hline Phenotype & Graul-Neumann et al ${ }^{8}$ & Horn and Robinson ${ }^{9}$ & Goldblatt et al ${ }^{10}$ & Takenouchi et all1 & Jacquinet et all2 & \multicolumn{2}{|c|}{ Garg and $X_{i n g}{ }^{13}$} \\
\hline Age (years) & 27 & 3.5 & 20 & 10 & 16 & 23 & 17 \\
\hline Gender & Female & Female & Male & Female & Female & Female & Female \\
\hline Birth weight (g) & 1780 & 1185 & 1040 & 1427 & 1720 & 1190 & 1172 \\
\hline Length (cm) & 41.5 & 40 & Not recorded & 40 & 45 & 40 & 40 \\
\hline Gestation & 36 weeks & 32 weeks & 28 weeks & 34 weeks & 39 weeks & 32 weeks & 32 weeks \\
\hline OFC $(\mathrm{cm})$ & 32 & 29 & Not recorded & 30.6 & 32 & 29 & 28.5 \\
\hline \multicolumn{8}{|l|}{ Marfanoid signs } \\
\hline Lens dislocation & Yes (13 years) & No & Yes (16 years) & No & No & Left eye & No \\
\hline Myopia & Severe & No & Severe & Severe & Severe & Yes & Yes \\
\hline Aortic root dilat & Yes & No & No & No & Yes & No & Yes \\
\hline Mitral valve prolapse & Yes & Yes & No & No & No & No & Yes \\
\hline Dural ectasia & Yes & Not recorded & Not recorded & Yes & Not recorded & \multicolumn{2}{|c|}{ Not recorded } \\
\hline Arachnodactyly & Yes & Yes & Yes & Yes & Yes & Yes & Yes \\
\hline Pectus excavatum & No & Not recorded & Yes & Yes & No & No & Yes \\
\hline Hyperextensible joints & Yes & Not recorded & Yes & Not recorded & Yes & Yes & Yes \\
\hline Scoliosis & No & Not recorded & No & No & No & Yes & Yes \\
\hline Progeroid facial signs & Yes & Yes & Yes & Yes & Yes & Yes & Yes \\
\hline Lipodystrophy congenital & Yes & Yes & Yes & Yes & Yes & Yes & Yes \\
\hline Glucose, insulin & Normal & Not recorded & Normal & Not recorded & Normal & \multicolumn{2}{|c|}{ Not recorded } \\
\hline
\end{tabular}

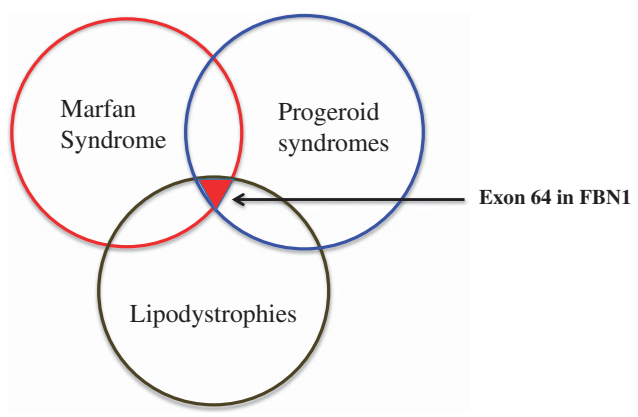

Figure 2 Overlapping clinical features in marfanoid-progeroid-lipodystrophy syndrome, resulting from mutations in exon 64 of the FBN1 gene.

the progeroid form of MFS. As not all mutations in exon 64 nor all premature truncation or splice site mutations in the $3^{\prime}$ exons of FBN1 are associated with the progeroid phenotype, ${ }^{7}$ the frameshift mutations described in the progeroid form of MFS must alter the function of fibrillin-1 in some specific way.

Further functional studies exploring the quantity and size of the fibrillins produced in this particular clinical context, as well as studies of the extracellular matrix composition are needed to better characterize the pathogenesis of this particular syndrome compared with other fibrillinopathies.

In summary, as specific mutations in the penultimate exon of the FBN1 gene result in similar clinical manifestations that overlap with those of MFS, progeroid syndro- mes, and lipodystrophies the designation marfanoid-progeroid-lipodystrophy syndrome appears to be appropriate for this newly defined genetic disorder.

\section{CONFLICT OF INTEREST}

The authors declare no conflict of interest.

\section{ACKNOWLEDGEMENTS}

We thank Mary F Passarge for critical reading of the manuscript and helpful suggestions.

\footnotetext{
1 Robinson P, Arteaga-Solis E, Baldock C et al: The molecular genetics of Marfan syndrome and related disorders. J Med Genet 2006; 43: 769-787.

2 Davis MR, Summers KM: Structure and function of the mammalian fibrillin gene family: implications for
}

human connective tissue diseases. Mol Genet Metab 2012; 107: 635-647.

3 De Paepe A, Devereux RB, Dietz HC et al: Revised diagnostic criteria for the Marfan syndrome. Am J Med Genet 1996; 62: 417-426.

4 Collod-Béroud G, Le Bourdelles S, Ades L et al: Update of the UMD-FBN1 mutation database and creation of an FBN1 polymorphism database. Hum Mutat 2003; 22: 199-208.

5 Stheneur C, Tubach F, Jouneaux M et al: Study of phenotype evolution during childhood in Marfan syndrome to improve clinical recognition. Genet Med 2014; 16: 246-250.

6 Faivre L, Collod-Beroud G, Loeys BL et al: Effect of mutation type and location on clinical outcome of 1,013 probands with Marfan syndrome or related phenotypes and FBN1 mutations: an international study. Am J Hum Genet 2007; 81: 454-466.

7 Schrijver I, Liu W, Odom R et al: Premature termination mutations in FBN1: distinct effects on differential allelic expression and on protein and clinical phenotypes. Am J Hum Genet 2002; 71: 223-237.

8 Graul-Neumann LM, Kienitz T, Robinson PN et al: Marfan syndrome with neonatal progeroid syndromelike lipodystrophy associated with a novel frameshift mutation at the 3' terminus of the FBN1 gene. Am J Med Genet A 2010; 152A: 2749-2755.

9 Horn D, Robinson PN: Progeroid facial features and lipodystrophy associated with a novel splice site mutation in the final intron of the FBN1 gene. Am J Med Genet A 2011; 155A: 721-724.

10 Goldblatt J, Hyatt J, Edwards C et al: Further evidence for a marfanoid syndrome with neonatal progeroid features and severe generalized lipodystrophy due to frameshift mutations near the 3' end of the FBN1 gene. Am J Med Genet A 2011; 155A: 717-720.

11 Takenouchi T, Hida M, Sakamoto $Y$ et al: Severe congenital lipodystrophy and a progeroid appearance: mutation in the penultimate exon of FBN1 causing a recognizable phenotype. Am J Med Genet A 2013; 161A: 3057-3062

12 Jacquinet $A$, Verloes $A$, Callewaert B et al: Neonatal progeroid variant of Marfan syndrome with congenital lipodystrophy results from mutations at the $3^{\prime}$ end of FBN1 gene. Europ J Med Genet 2014; 57: 230-234.

13 Garg A, Xing C: De Novo heterozygous FBN1 mutations in the extreme $\mathrm{C}$-terminal region cause progeroid 
fibrillinopathy. Am J Med Genet Part A 2014; 164A: 1341-1345.

14 Sakai LV, Keane DR, Engvall E: Fibrillin, a new 350-kD glycoprotein, is a major component of extracellular microfibrils. J Cell Biol 1986; 103: 2499-2509.

15 Raghunath M, Putnam EA, Ritty $T$ et al: Carboxyterminal conversion of profibrillin to fibrillin at a basic site by PACE/furin-like activity required for incorporation in the matrix. J Cell Sci 1999; 112: 1093-1100.

16 Hubmacher D, Reinhardt DP: One more piece in the fibrillin puzzle. Structure 2009; 17: 635-636.

17 Jensen SA, Robertson IB, Handford PA: Dissecting the fibrillin microfibril: structural insights into organization and function. Structure 2012; 20: 215-225.
18 Piha-Gossack A, Sossin W, Dieter P, Reinhardt DP: The evolution of extracellular fibrillins and their functional domains. PLoS One 2012; 7: e33560.

19 Giltay R, Timpl R, Kostka G: Sequence, recombinant expression and tissue localization of two novel extracellular matrix proteins, fibulin-3 and fibulin-4. Matrix Biol 1999; 18: 469-480. 